\title{
Communication \\ Orbital Angular Momentum Mode Sensing Technology Based on Intensity Interrogation
}

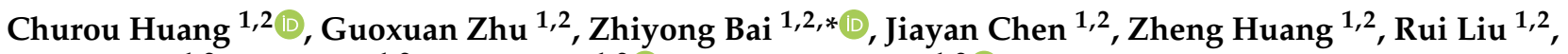 \\ Luping $\mathrm{Wu}^{1,2}$, Shen Liu ${ }^{1,2}$, Cailing Fu ${ }^{1,2}$ and Yiping Wang ${ }^{1,2}$ (D)
}

1 Key Laboratory of Optoelectronic Devices and Systems of Ministry of Education/GuangDong Province, College of Physics and Optoelectronic Engineering, Shenzhen University, Shenzhen 518060, China; 1900453003@email.szu.edu.cn (C.H.); zhugxuan@szu.edu.cn (G.Z.); 1900453055@email.szu.edu.cn (J.C.); 1910454042@email.szu.edu.cn (Z.H.); 2060453053@email.szu.edu.cn (R.L.); 2070456106@email.szu.edu.cn (L.W.); shenliu@szu.edu.cn (S.L.); fucailing@szu.edu.cn (C.F.); ypwang@szu.edu.cn (Y.W.)

2 Shenzhen Key Laboratory of Photonic Devices and Sensing Systems for Internet of Things, Guangdong and Hong Kong Joint Research Centre for Optical Fibre Sensors, Shenzhen University, Shenzhen 518060, China

* Correspondence: baizhiyong@szu.edu.cn

Citation: Huang, C.; Zhu, G.; Bai, Z.; Chen, J.; Huang, Z.; Liu, R.; Wu, L.; Liu, S.; Fu, C.; Wang, Y. Orbital Angular Momentum Mode Sensing Technology Based on Intensity Interrogation. Sensors 2022, 22, 1810 https://doi.org/10.3390/s22051810

Received: 30 December 2021

Accepted: 24 February 2022

Published: 25 February 2022

Publisher's Note: MDPI stays neutral with regard to jurisdictional claims in published maps and institutional affiliations.

Copyright: (C) 2022 by the authors. Licensee MDPI, Basel, Switzerland. This article is an open access article distributed under the terms and conditions of the Creative Commons Attribution (CC BY) license (https:// creativecommons.org/licenses/by/ $4.0 /)$.

\begin{abstract}
A novel optical fiber sensing technology based on intensity distribution change in orbital angular momentum (OAM) mode is proposed and implemented herein. The technology utilizes a chiral long-period fiber grating (CLPFG) to directly excite the 1st-order OAM $\left(\mathrm{OAM}_{1}\right)$ mode. The intensity changes in the coherent superposition state between the fundamental mode and the $\mathrm{OAM}_{1}$ mode at the non-resonant wavelength of the CLPFG is tracked in order to sense the external parameters applied to the grating area. Applying this technology to temperature measurement, the intensity distribution change has a good linear relationship with respect to temperature in the range of $30^{\circ} \mathrm{C}$ to $100{ }^{\circ} \mathrm{C}$. When the intensity was denoted by the number of pixels with a gray value of one after binarization of collected images, the sensitivity was $103 \mathrm{px} /{ }^{\circ} \mathrm{C}$ and the corresponding resolution was $0.0097^{\circ} \mathrm{C}$. Meanwhile, theoretical and experimental results show that the sensitivity and resolution can be further improved via changing the area of the collected image. Compared with sensing methods based on spiral interference pattern rotation in previous work, this sensing technology has the advantage of exquisite structure, easy realization, and good stability, thus making it a potential application in practices.
\end{abstract}

Keywords: orbital angular momentum; chiral long-period fiber grating; temperature sensor; intensity interrogation

\section{Introduction}

Orbital angular momentum (OAM) beam [1] is a kind of beam with a helical phase front, which is generally expressed by $\exp (\mathrm{il} \varphi)$, where 1 represents the topological charge number and $\varphi$ is the azimuth angle. This special phase structure induces a phase singularity, and a donut-shaped intensity profile of an OAM beam. Due to the unique structure, the OAM beam has been widely utilized in optical communications [2,3], optical manipulation [4], optical imaging [5], and especially in optical sensing. For instance, the OAM beams have been used to measure the rotation of an object based on Doppler shift without the need for complicated object image reconstruction [6], and the OAM-based technology [7] expands a new optical degree of freedom perspective to detect the lateral motion in an arbitrary direction. Also, magnetic field measurement [8] applies OAM modes with opposite propagating directions that interfere to form petal-shaped images. It turns out that the rotation angle of the petal-shaped images changes with the change in the magnetic field and the sensitivity is $28^{\circ} / \mathrm{T}$. The method is suitable for high-precision measurement applications, however, a higher cost and a larger volume spatial OAM generator was used 
in the experiment. Recently, optical fiber sensors based on OAM modes [9] have been proposed and achieved by tracking the rotation angle of the spiral interference patterns resulting from the co-axis interference between OAM modes and Gaussian beams. The sensitivity of temperature is $87.09^{\circ} /{ }^{\circ} \mathrm{C}$. The advantage of the OAM-based fiber sensor is mainly to measure the changes in the external parameters through the rotation angle of spiral interference fringes, which avoids the reading error of non-integer fringes in the traditional interference measurement. The sensitivity and resolution are generally high. At present, optical fiber sensors based on OAM mainly use the structure of double optical path interference. The OAM sensing technology based on the interference of the Gaussian beam and the OAM beam has been reported in literature [10-12]. The phase difference of the two optical paths is changed by heating the sensing fiber, resulting in the rotation of the spiral interference pattern. The temperature change is tracked by monitoring the rotation angle of the spiral interference pattern. The difference is that their OAM generators and demodulation methods are different. The OAM generators are a hologram [10], a spiral phase plate [11], and long-period fiber grating and a rotator [12] respectively. The disadvantages of this structure are that the interference pattern is easily affected by external parameters, is unstable, and is difficult to collect, which has an adverse impact on its practical application.

In this paper, a single optical path sensing technique is proposed by tracking the intensity change in the superimposed state between the fundamental mode $\left(\mathrm{OAM}_{0}\right)$ and the 1 storder OAM $\left(\mathrm{OAM}_{1}\right)$ mode. The single optical path was composed by a chiral long-period fiber grating (CLPFG) and a section of two-mode fiber (TMF) [10]. The CLPFG [11-16] was used to directly excite the $\mathrm{OAM}_{1}$ mode. At the non-resonant wavelength of CLPFG, the $\mathrm{OAM}_{0}$, and $\mathrm{OAM}_{1}$ mode coexisted and then were transmitted in TMF. At the output end of TMF, the coherent superimposition of the two modes was obtained. When the environmental variables were applied to the CLPFG, the energy ratio between the $\mathrm{OAM}_{0}$ and $\mathrm{OAM}_{1}$ mode changed and then influenced the intensity of the superimposed mode. The sensing technology was applied in the temperature measurement and the consequence shows that the sensitivity and the resolution are $103 \mathrm{px} /{ }^{\circ} \mathrm{C}$ and $0.0097{ }^{\circ} \mathrm{C}$ in the range of $30{ }^{\circ} \mathrm{C}$ to $100{ }^{\circ} \mathrm{C}$, respectively. Meanwhile, the linear fitting results show that the intensity change has a linear relationship with temperature applied. By changing the image area, the sensitivity can be adjusted from $1 \mathrm{px} /{ }^{\circ} \mathrm{C}$ to $103 \mathrm{px} /{ }^{\circ} \mathrm{C}$.

\section{Principle}

The schematic diagram of the operation principle is shown in Figure 1. The sensing system is composed of an OAM mode generator and a section of TMF. In this work, the OAM mode generator is a CLFPG, which was fabricated by twisting molten single-mode fiber (SMF-28e) at high temperatures. As illustrated in the inset of Figure 1, when the grating pitch of the CLPFG is $530 \mu \mathrm{m}$, the resonant wavelength is $1572.5 \mathrm{~nm}$ and the dip loss is $31.2 \mathrm{~dB}$, thus directly coupling the $\mathrm{OAM}_{0}$ to the $\mathrm{OAM}_{1}$ mode with high efficiency. When the incident light is located at the resonant wavelength of CLPFG, the fundamental mode of the fiber, that is, the $\mathrm{OAM}_{0}$ mode, is completely transformed into the $\mathrm{OAM}_{1}$ mode by the CLFPG. As shown in Figure 2, it can be calculated, by finite element analysis, that TMF (14 $\mu \mathrm{m} / 125 \mu \mathrm{m}$ for core/cladding diameter) can support the vector modes of $\mathrm{HE}_{21}$ even, $\mathrm{HM}_{21}$ odd, $\mathrm{TE}_{01}$, and $\mathrm{TM}_{01}$, which can be superimposed into the $\mathrm{OAM}_{1}$ mode. Therefore, the TMF is fused at the back end of CLFPG, which can transmit the OAM mode excited in CLPFG within a certain distance. At the back end of the TMF, only the ring-shape mode profile belonging to the $\mathrm{OAM}_{1}$ mode can be seen. When the wavelength of the input light is far from the grating loss dip, the $\mathrm{OAM}_{0}$ mode can be seen at the back end of TMF. When the input light has a wavelength within the loss dip bandwidth, a part of the $\mathrm{OAM}_{0}$ mode energy is coupled to the OAM $\mathrm{OM}_{1}$ mode. At this time, the $\mathrm{OAM}_{0}$ and $\mathrm{OAM}_{1}$ mode coexist. After they are transmitted through the TMF, mode interference occurs at the output end, and the coherent superposition state of the two modes is observed. 


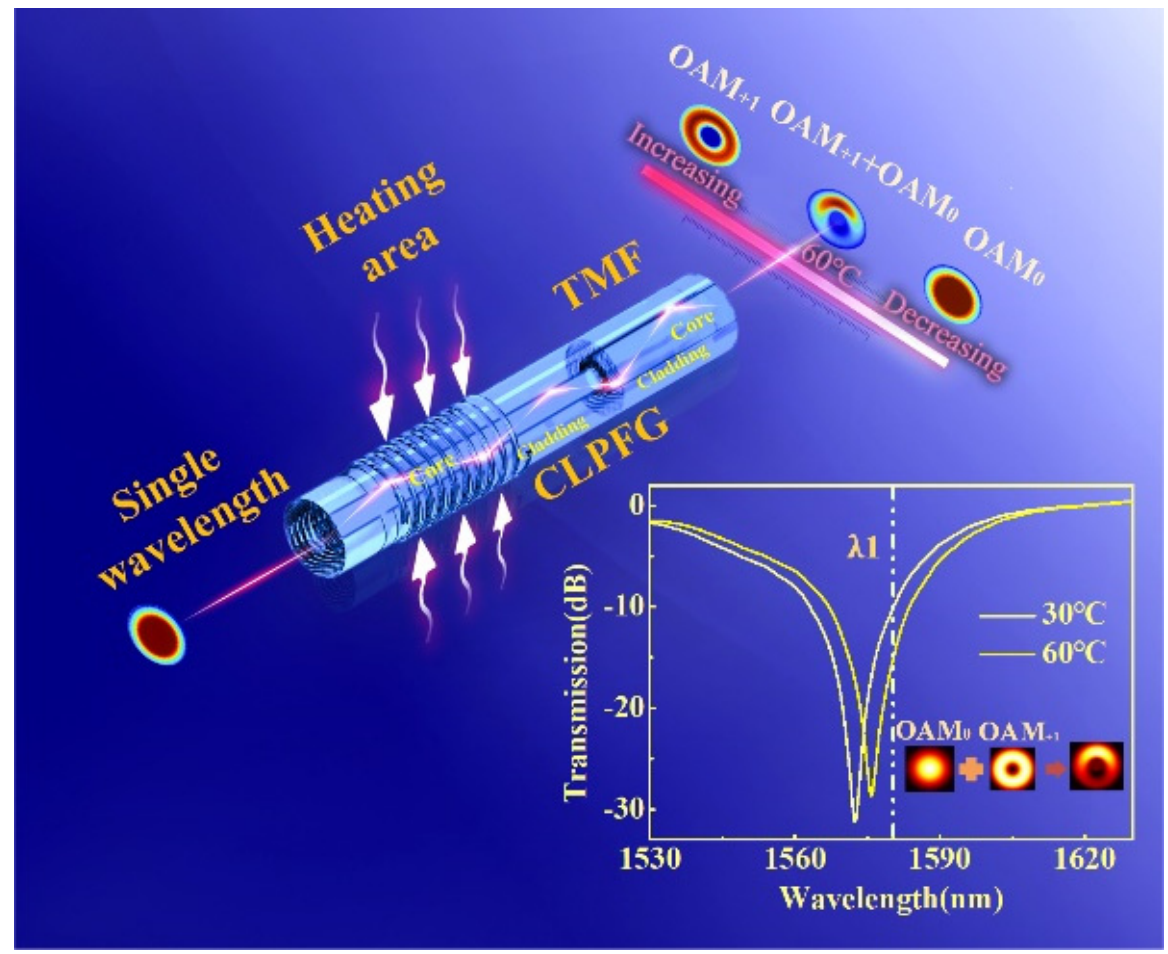

Figure 1. Schematic diagram of sensing principle. TMF: two-mode fiber; the inset is the transmission spectrum of CLPFG at a different temperature.

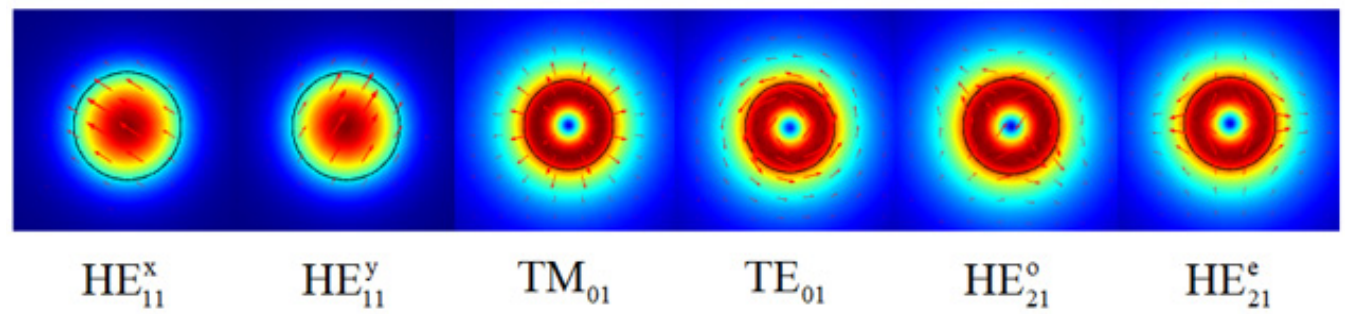

Figure 2. Schematic diagram of TMF transmission mode obtained by simulation.

Generally speaking, by changing the external environment parameters of the TMF, the phase difference between the $\mathrm{OAM}_{0}$ and $\mathrm{OAM}_{1}$ mode can be changed, the rotation change in the superposition state can be observed at the output end, and then the sensing measurement can be carried out by tracking the rotation angle. However, the OAM mode generator is an all-fiber device. It is difficult to achieve perfect coaxially distribution in the intensity of the OAM mode. Therefore, when the sensor is performed by tracking the phase shift, except the rotation of the spiral interference pattern, the intensity distribution is changed as well, which brings potential difficulties for accurate image acquisition and accurate measurement. Although the sensing sensitivity is high, it is difficult to achieve the stable acquisition and processing of rotating images, which limits its practical application. Thus, the measurement can be realized by tracking the intensity change in the interference patterns, where the spiral interference patterns only changes in intensity without rotation. Taking temperature as an example, when the external temperature changes, the grating refractive index changes due to the thermal effect and the thermal expansion effect, resulting in the shift of resonant wavelength. Thus, coherent superposition state between the $\mathrm{OAM}_{0}$ and $\mathrm{OAM}_{1}$ mode is observed in CLPFG at the same wavelength near the resonant wavelength. Different temperatures affect the change in energy ratio. In this paper, by applying variable environmental parameters to the OAM mode generator, that is the CLPFG, the change in the energy proportion of the $\mathrm{OAM}_{0}$ and $\mathrm{OAM}_{1}$ mode can be induced, due to the response 
characteristics of the CLPFG. In this work, the measurement of environmental parameters can be realized via tracking the change in the intensity profile of the superimposed modes.

The inset in Figure 1 shows the transmission spectrum of CLPFG, from which the $\mathrm{OAM}_{0}$ and $\mathrm{OAM}_{1}$ mode coexist at the non-resonant wavelength $\lambda_{1}$. The temperature measurement is taken as an example to illustrate the sensing and interrogating technology. When the temperature changes, the energy ratio of the $\mathrm{OAM}_{0}$ and $\mathrm{OAM}_{1}$ mode changes, resulting in a change in the intensity distribution of the superposition state. As is shown in Figure 3, the numerical calculation of sensing principle can be obtained. First, the $\mathrm{OAM}_{1}$ mode measured at the resonant wavelength and the $\mathrm{OAM}_{0}$ mode measured without writing CLPFG are regarded as the calculation basis. Second, by changing the energy ratio of the two modes, the intensity changes in the superposition state can be calculated, as shown in Figure 3a. In the calculation process, the superimposed mode field is binarized, and the total number of pixels with a gray value of one is used to represent the intensity. Then, the change in intensity, with respect to energy ratio, is fitted and plotted in Figure $3 b$, and the sensitivity and linearity $\left(\mathrm{R}^{2}\right)$ are predicted. It is worth noting that the size of the acquired image and the setting of the threshold in the binarization process will affect the results. As shown in Figure 3c, S1-S6 represent different imaging areas, respectively, as follows: $0.41 \mathrm{~mm}^{2}, 1.21 \mathrm{~mm}^{2}, 3.27 \mathrm{~mm}^{2}, 6.88 \mathrm{~mm}^{2}, 11.58 \mathrm{~mm}^{2}$, and $18.69 \mathrm{~mm}^{2}$. When the threshold is greater than 10 (grayscale value 0-255), the sensitivity and linearity of the intensity-to-energy ratio response tend to be stable under different imaging areas, indicating that an appropriate threshold can eliminate the influence of background noise. At this time, the corresponding threshold can be determined. For different imaging areas, the sensitivity is different. Figure $3 \mathrm{~d}$ redraws the sensitivity of the different imaging areas at a threshold of 17 . The sensitivity gradually increases as the imaging area increases.

(a)

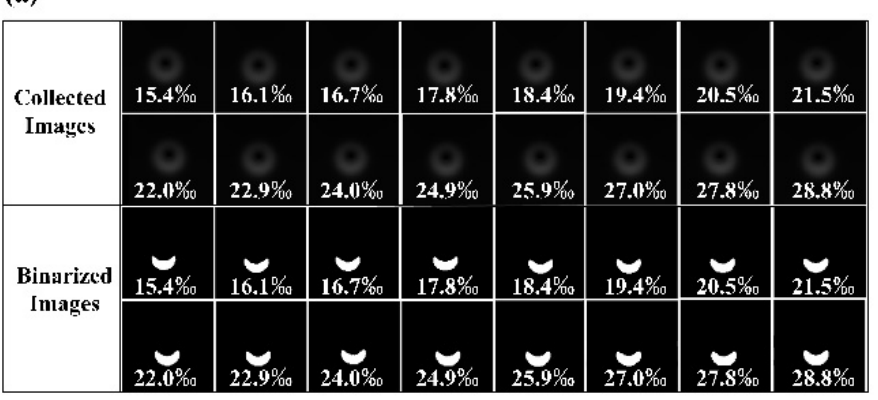

(c)

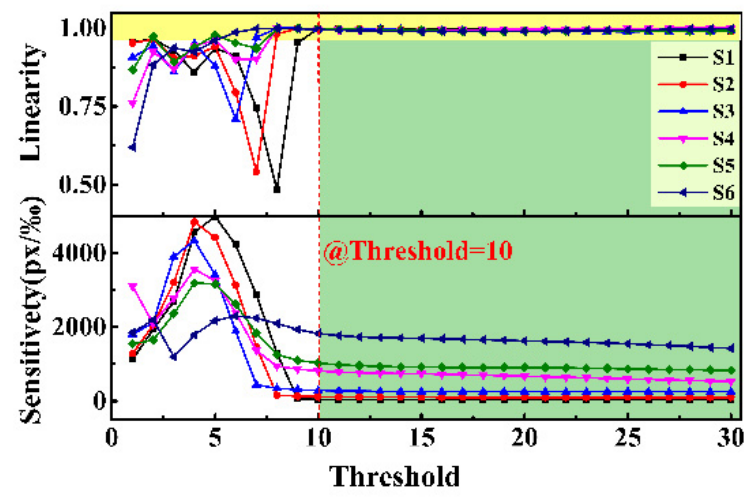

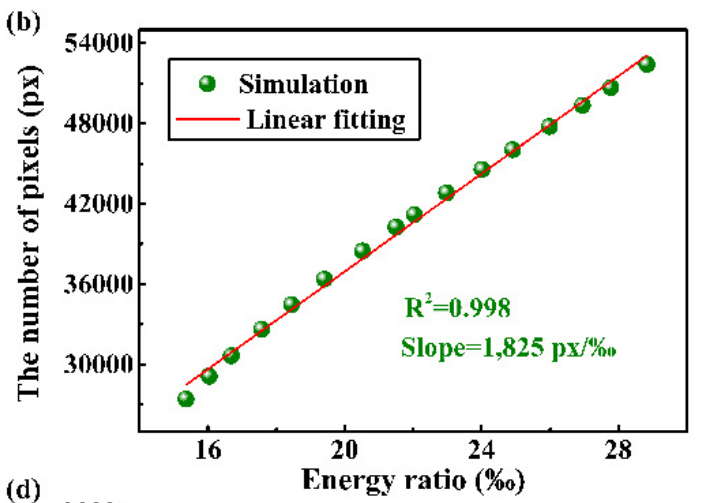

(d)

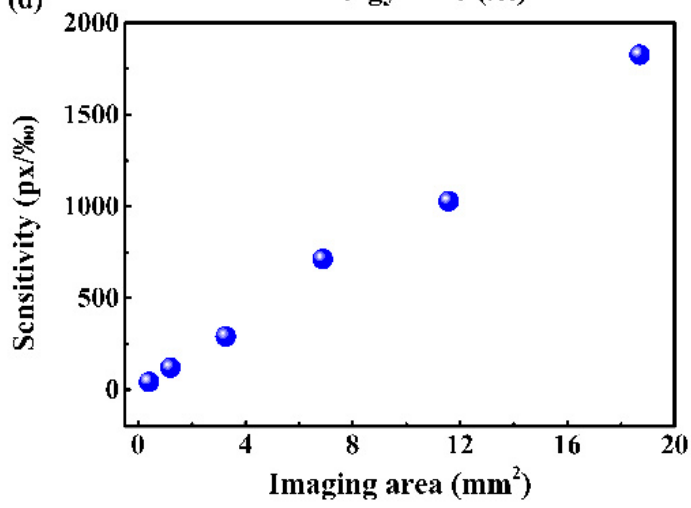

Figure 3. Numerical calculation of sensing principle. (a) The collected and binarized images of the intensity distributions in different energy ratio between the $\mathrm{OAM}_{0}$ and $\mathrm{OAM}_{1}$ mode; (b) the relationship between the energy ratio and the number of pixels with the gray value of 1 ; (c) the evolution of sensitivity and linearity of the sensor with the changes in imaging area and thresholds, and the size of imaging areas denoted by S1-S6 are $0.41 \mathrm{~mm}^{2}, 1.21 \mathrm{~mm}^{2}, 3.27 \mathrm{~mm}^{2}, 6.88 \mathrm{~mm}^{2}$, $11.58 \mathrm{~mm}^{2}$, and $18.69 \mathrm{~mm}^{2}$, respectively; (d) the relationship between sensitivity and image area. 


\section{Sensing Characteristics}

The experimental setup of the proposed sensing method is shown in Figure 4, which is mainly composed of a tunable laser emitting single-wavelength light, a CLPFG that excites the $\mathrm{OAM}_{1}$ mode, and a CCD that collects the intensity distribution. The tunable laser (Agilent $81940 \mathrm{~A}, \mathrm{USA}$ ) has relatively stable power while emitting. When the output laser exceeded 1 hour, the power fluctuation range was $\pm 0.0075 \mathrm{~dB}$, which can be negligible. The CLFPG, with a resonant wavelength of $1572.5 \mathrm{~nm}$, was fabricated by twisting the hot-melt SMF. As such, a section of TMF was fused at the end of the grating at an interval of $5 \mathrm{~mm}$ to transmit high-order modes in order to facilitate the measurement of the mode field distribution and its changes. We used a compact precision temperature control box (LCO 102 SINGLE, ECOM) as the temperature generator. The device can produce temperature changes in the range of $0-100{ }^{\circ} \mathrm{C}$, with an accuracy of $0.01{ }^{\circ} \mathrm{C}$. The CLPFG of the measuring section was placed in a closed temperature control box, and both ends of the optical fiber passing through the temperature control box were fixed on the optical fiber clamp. Through heat conduction, CLPFG can obtain the same temperature change as the temperature control box, so as to study the temperature sensing performance of the sensor. The operation wavelength was selected to be $1570.5 \mathrm{~nm}$, in which the $\mathrm{OAM}_{0}$ and $\mathrm{OAM}_{1}$ mode were in the state of coexistence. It cannot be ignored that all-optical fiber devices are fixed on the platform, which helps to collect the stable images. The temperature rose from $30{ }^{\circ} \mathrm{C}$ to $100^{\circ} \mathrm{C}$, at an interval of $5{ }^{\circ} \mathrm{C}$, and each temperature remained for $5 \mathrm{~min}$ in order to ensure that the grating was in a state of constant temperature. The CCD (Hamamatsu, C12741-03, Japan) has a resolution of $640 \times 512 \mathrm{px}$ and the size of the single-pixel is $20 \mu \mathrm{m} \times 20 \mu \mathrm{m}$. It recorded the changes in the superimposed mode intensity. According to the display, the intensity of the images must not be exposed, so as to achieve a good match between the intensity distribution and the gray value of images. What's more, the type of images collected by the camera is uint8, which has a gray-level range of 0-255.

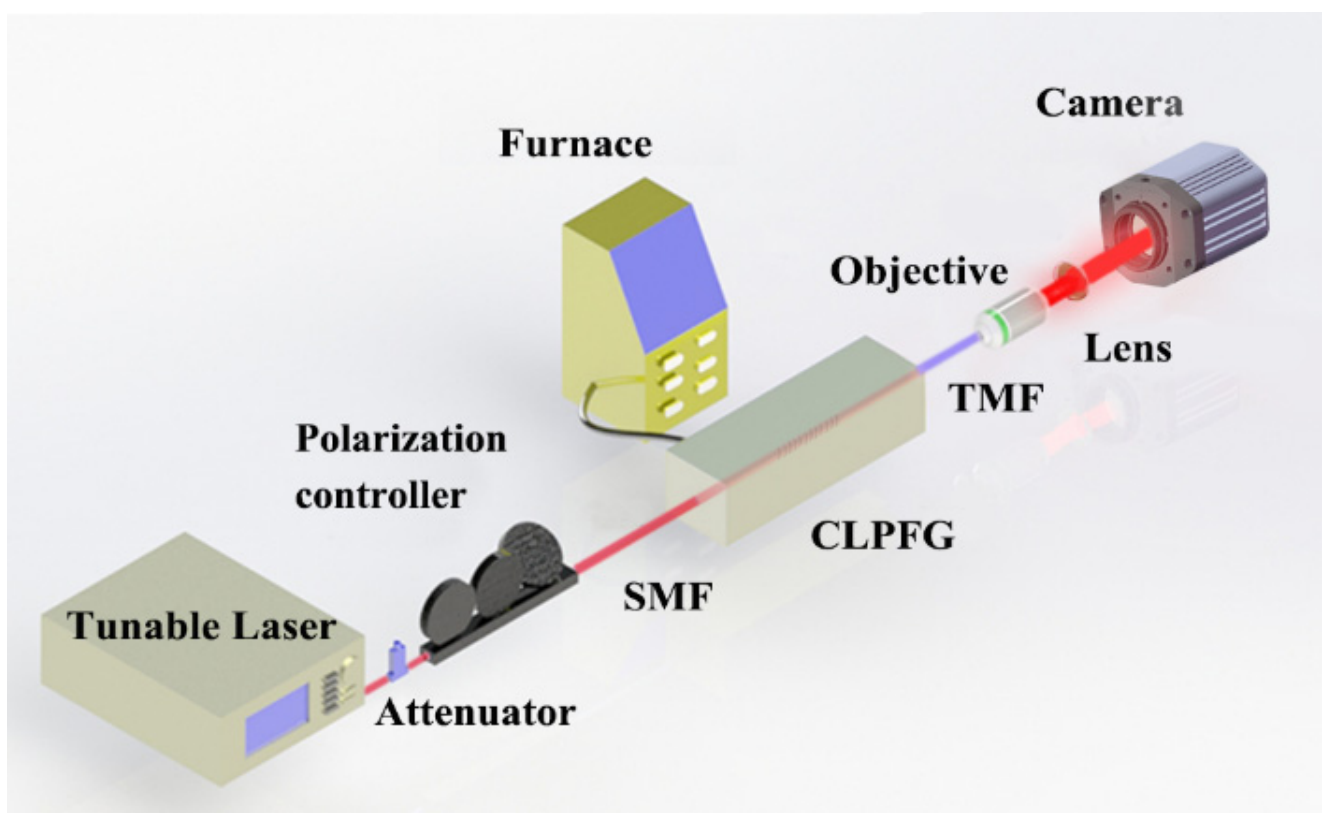

Figure 4. The experiment system of temperature measurement.

The detailed experiment of temperature measurement was performed as follows. First, the intensity distribution of the coherent superposition modes, with an imaging area of $7.83 \mathrm{~mm}^{2}$, were collected when the temperature increased from $30^{\circ} \mathrm{C}$ to $100^{\circ} \mathrm{C}$. Second, the collected images were binarized under the threshold with a gray value of 41 . The collected images and their corresponding binarized results are shown in Figure 5a. Third, the number of pixels with a gray value of one, representing intensity information, was 
counted and is plotted here in Figure 5b. And then, we repeated the measurement step for the cooling process, and plotted the intensity distribution pattern and experiment data in Figure 5a,b respectively. In Figure 5a, the intensity distribution pattern of the interference pattern does not rotate, and only shows the change in intensity. In Figure $5 b$, the intensity change data measured during the heating and cooling show linear monotonicity. Through fitting, the temperature sensitivity and linearity under the test conditions are $48 \mathrm{px} /{ }^{\circ} \mathrm{C}$ and 0.995 , respectively.

(a)

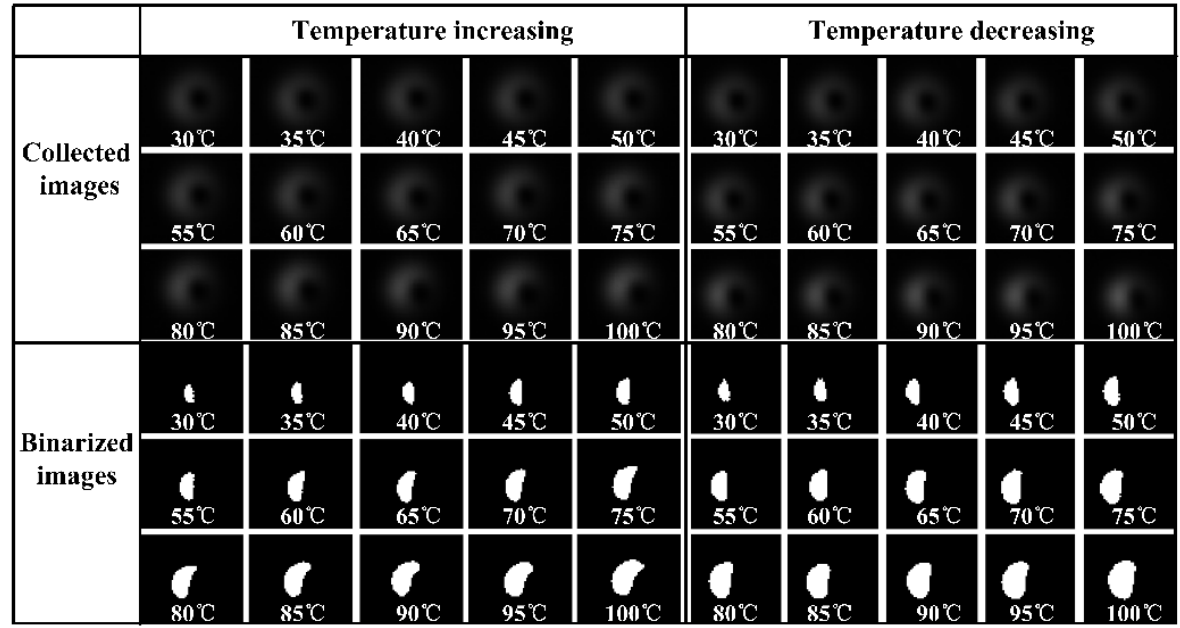

(b)

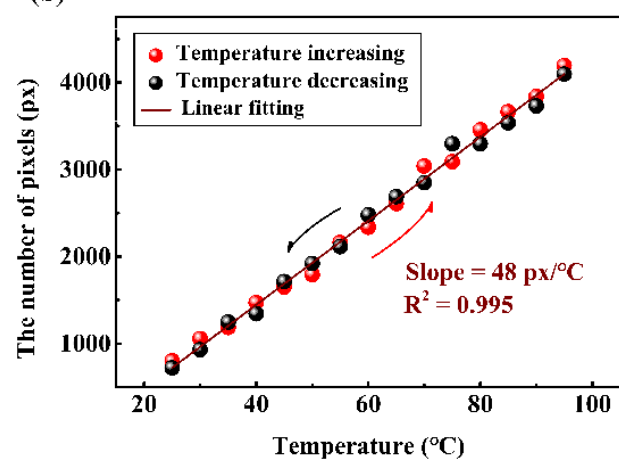

(c)

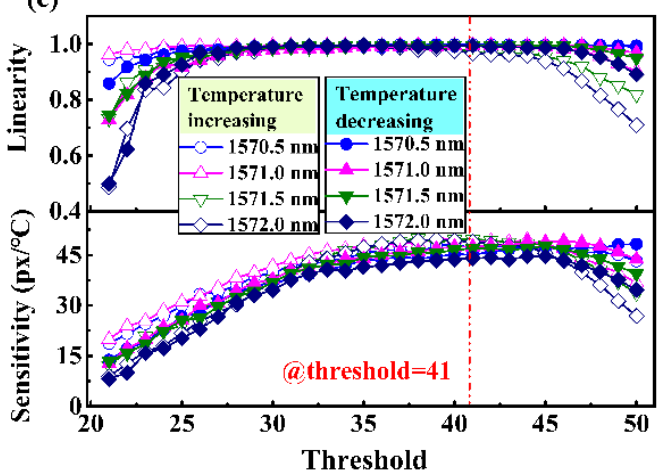

Figure 5. Experimental measurement results. (a-c) Experiment data with imaging area $7.83 \mathrm{~mm}^{2}$ with temperature increasing and decreasing: (a) the collected and binarized images of the intensity distributions from $30{ }^{\circ} \mathrm{C}$ to $100{ }^{\circ} \mathrm{C}$ at $1571 \mathrm{~nm}$; (b) experiment data of the temperature test and its linear fitting; (c) the evolution of sensitivity and linearity with different operation wavelength and threshold.

In the process of temperature detection, different operating wavelengths and different binarization thresholds affect the sensitivity of the test. Therefore, the effects of these two factors on the sensitivity were studied. We repeated the temperature measurement processing and scanned the operation wavelength from $1560 \mathrm{~nm}$ to $1580 \mathrm{~nm}$, with an interval of $0.5 \mathrm{~nm}$. The recorded intensity graphs of the superimposed modes were binarized with the threshold from 20 to 50 . The variation trend of the measured sensitivity with wavelength and threshold is shown in Figure 5c. The temperature response sensitivity generally shows a trend of first increasing and then decreasing, for which, as the operation wavelength is close to the resonant wavelength, the slope of the energy ratio first increases and then decreases. In the meantime, due to the defects in the collected images, the linearity of the different wavelengths under the different thresholds fluctuates. Therefore, choosing an appropriate threshold can improve the stability and accuracy of the measurement. Generally speaking, when the threshold is 41 , the temperature sensitivity at the wavelength of $1571 \mathrm{~nm}$ is higher and the linearity is also improved, respectively, about $48 \mathrm{px} /{ }^{\circ} \mathrm{C}$ and 0.995 . 
In theory, the sensitivity can be enhanced by changing the number of participating pixels. Thus, we studied the evolution of sensitivity under different imaging areas. The intensity distributions shown in Figure 6a, represent the different imaging areas of $0.15 \mathrm{~mm}^{2}$, $0.85 \mathrm{~mm}^{2}, 2.31 \mathrm{~mm}^{2}, 3.76 \mathrm{~mm}^{2}, 7.83 \mathrm{~mm}^{2}, 9.26 \mathrm{~mm}^{2}$, and $16.38 \mathrm{~mm}^{2}$, respectively. In Figure $6 \mathrm{~b}$, the corresponding sensitivities are $1 \mathrm{px} /{ }^{\circ} \mathrm{C}, 5 \mathrm{px} /{ }^{\circ} \mathrm{C}, 14 \mathrm{px} /{ }^{\circ} \mathrm{C}, 27 \mathrm{px} /{ }^{\circ} \mathrm{C}$, $48 \mathrm{px} /{ }^{\circ} \mathrm{C}, 53 \mathrm{px} /{ }^{\circ} \mathrm{C}$, and $103 \mathrm{px} /{ }^{\circ} \mathrm{C}$, respectively. When the imaging area was expanded to $16.38 \mathrm{~mm}^{2}$ within the limited experiment condition, we repeated the experiment setup above. The corresponding results are shown in Figure 6c. The temperature sensitivity $103 \mathrm{px} /{ }^{\circ} \mathrm{C}$ was achieved at a threshold of 60 , which is larger than the sensitivity of the imaging area of $7.83 \mathrm{~mm}^{2}$. Since the CCD can recognize one pixel at least, the theoretical temperature resolution of the sensing technology is $0.0097^{\circ} \mathrm{C}$. In addition, according to the method obtained in Figure 5c, we explored the evolution process of the different working wavelengths under the different thresholds in the imaging area of $16.38 \mathrm{~mm}^{2}$, as shown in Figure 6d. By optimizing the optical aperture of the imaging element, the highest sensitivity (of about $809 \mathrm{px} /{ }^{\circ} \mathrm{C}$ ) may be obtained by further enlarging the imaging area. In the experiment, the sensitivity can be improved from $1 \mathrm{px} /{ }^{\circ} \mathrm{C}$ to $103 \mathrm{px} /{ }^{\circ} \mathrm{C}$, under the current system conditions.

(a)

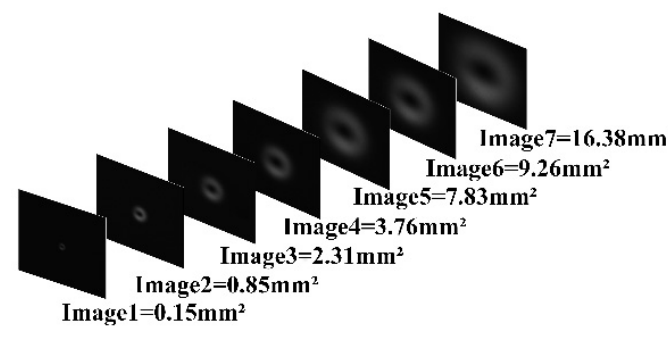

(c)

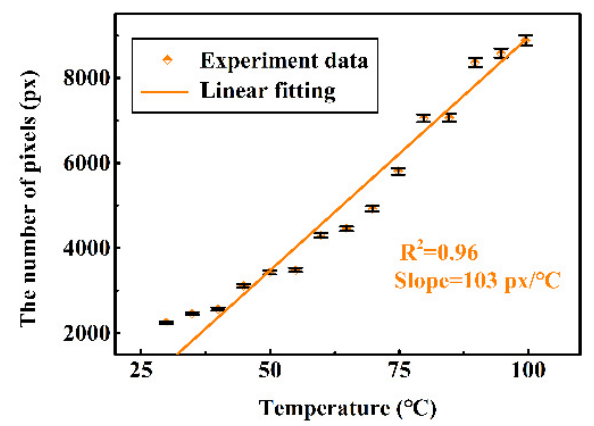

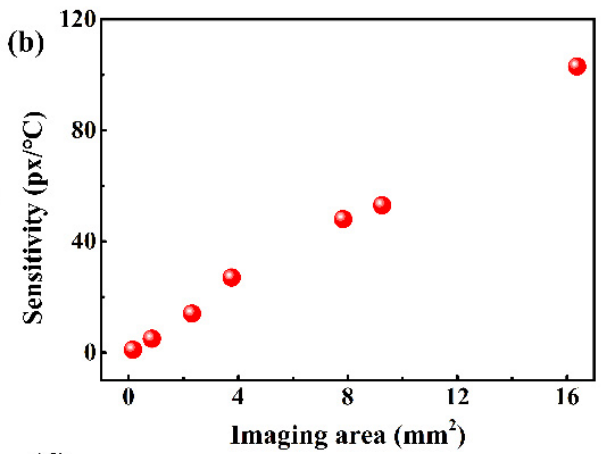

(d)

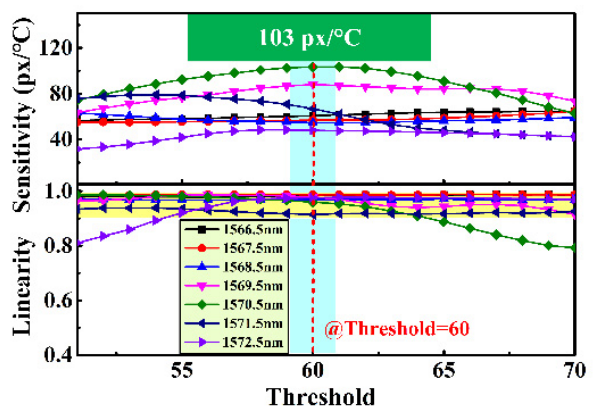

Figure 6. (a) The collected images with different imaging area; (b) the sensitivity changes with respect to the different imaging area. (c,d) Experiment data with imaging area $16.38 \mathrm{~mm}^{2}$ in temperature increasing: (c) experiment data of temperature test and its linear fitting; (d) the evolution of sensitivity and linearity with different operation wavelength and threshold.

At present, the representatives of fiber-based temperature measurement technology include wavelength demodulation technology and intensity demodulation technology. The general wavelength demodulation technology has a sensitivity of $132.8 \mathrm{pm} /{ }^{\circ} \mathrm{C}$ [17] and a resolution of about $0.1^{\circ} \mathrm{C}$. The temperature demodulation technology based on intensity [18] has low sensing resolution, i.e., $0.5^{\circ} \mathrm{C}$. However, the theoretical resolution of the temperature sensor based on OAM [9] can reach $10^{-7}{ }^{\circ} \mathrm{C}$, and the actual measurement can reach $10^{-5}{ }^{\circ} \mathrm{C}$. The temperature sensing technology based on intensity demodulation of OAM interference that is proposed in this work has better stability and is more easily applied, although its sensitivity is lower than that of the phase-shift OAM-based sensor. 


\section{Conclusions}

An optical fiber sensing technology based on intensity distribution change in OAM mode have been investigated and verified. The results display that the response of energy change to temperature is linear, with a sensitivity of $103 \mathrm{px} /{ }^{\circ} \mathrm{C}$ and a resolution of $0.0097^{\circ} \mathrm{C}$. Different operation wavelengths have different sensitivities, which provides a method to adjust the sensitivity. Moreover, the larger the imaging area, the higher the sensitivity and resolution of the sensing system. By using a CCD with higher resolution and increasing the imaging area, the sensitivity and resolution can be improved. This method has a simple structure, small size, high stability, and it has better application potential. In practical application, because CLPFG has a response to a variety of parameters, it brings the problem of cross sensitivity, which can be solved by studying the multi-parameter simultaneous measurement technology based on mode profile change.

Author Contributions: Conceptualization, C.H. and G.Z.; experimental methodology, C.H. and J.C.; software, C.H. and Z.H.; figures plotting, R.L. and L.W.; writing—original draft preparation, C.H.; supervision, S.L. and C.F.; writing-review and editing, Z.B. and Y.W. All authors have read and agreed to the published version of the manuscript.

Funding: This research was funded by National Natural Science Foundation of China, grant number 61875134, 62005169, 61905155; Shenzhen Science and Technology Innovation Commission, grant number JCYJ20180507182058432; Shenzhen University, grant number 2019097; Foundation for Distinguished Young Talents in Higher Education of Guangdong, China, grant number 2018KQNCX219.

Institutional Review Board Statement: Not applicable.

Informed Consent Statement: Not applicable.

Data Availability Statement: Data underlying the results presented in this paper are not publicly available at this time but may be obtained from the authors upon reasonable request.

Conflicts of Interest: The authors declare no conflict of interest.

\section{References}

1. Allen, L.; Beijersbergen, M.W.; Spreeuw, R.J.C.; Woerdman, J.P. Orbital angular momentum of light and the transformation of Laguerre-Gaussian laser modes. Phys. Rev. A 1992, 45, 8185-8189. [CrossRef] [PubMed]

2. Lavery, M.P.J.; Tur, M.; Ramachandran, S.; Molisch, A.F.; Ashrafi, N.; Ashrafi, S. Optical communications using orbital angular momentum beams. Adv. Opt. Photonics 2015, 7, 66-106. [CrossRef]

3. Yuan, Y.; Lei, T.; Li, Z.; Li, Y.; Gao, S.; Xie, Z.; Yuan, X. Beam wander relieved orbital angular momentum communication in turbulent atmosphere using Bessel beams. Sci. Rep. 2017, 7, srep42276. [CrossRef] [PubMed]

4. Shen, Y.; Wang, X.; Xie, Z.; Min, C.; Fu, X.; Liu, Q.; Gong, M.; Yuan, X. Optical vortices 30 years on: OAM manipulation from topological charge to multiple singularities. Light Sci. Appl. 2019, 8, 90. [CrossRef] [PubMed]

5. Yao, A.M.; Padgett, M.J. Orbital angular momentum: Origins, behavior and applications. Adv. Opt. Photonics 2011, 3, 161-204. [CrossRef]

6. Wisniewski-Barker, E.; Gibson, G.M.; Gibson Franke-Arnold, S.; Boyd, R.W.; Padgett, M.J. Mechanical Faraday effect for orbital angular momentum-carrying beams. Opt. Express 2014, 22, 11690-11697. [CrossRef] [PubMed]

7. Cvijetic, N.; Milione, G.; Ip, E.; Wang, T. Detecting Lateral Motion using Light's Orbital Angular Momentum. Sci. Rep. 2015, 5, 15422. [CrossRef] [PubMed]

8. Yu, S.; Pang, F.; Liu, H.; Li, X.; Yang, J.; Wang, T. Compositing orbital angular momentum beams in $\mathrm{Bi}_{4} \mathrm{Ge}_{3} \mathrm{O}_{12}$ crystal for magnetic field sensing. Appl. Phys. Lett. 2017, 111, 091107. [CrossRef]

9. Fu, H.; Wang, S.; Chang, H.; You, Y. A high resolution and large range fiber Bragg grating temperature sensor with vortex beams. Opt. Fiber Technol. 2020, 60, 102369. [CrossRef]

10. Soskin, M.S.; Alexeyev, A.N.; Volyar, A.V.; Izdebskaya, Y.V.; Soskin, M.S.; Vasnetsov, M.V. Fiber optical interferometric sensors of physical values with a singular reference beam in Second International Conference on Singular Optics (Optical Vortices). Fundam. Appl. 2001, 4403, 263-265.

11. Qiu, L.-Q.; Hu, H.-F.; Zhao, Y.; Li, J.; Wang, Q. Fiber optic temperature sensor using the orbital angular momentum and gaussian beams. Instrum. Sci. Technol. 2016, 45, 123-136. [CrossRef]

12. Gospodaric, J.; Kuzmenko, A.; Pimenov, A.; Huber, C.; Suess, D.; Rotter, S.; Pimenov, A. Optical fiber sensing technology based on Mach-Zehnder interferometer and orbital angular momentum beam. Appl. Phys. Lett. 2018, 112, 221105.

13. Zhao, Y.H.; Liu, Y.Q.; Zhang, L.; Zhang, C.Y.; Wen, J.X.; Wang, T.Y. Mode converter based on the long-period fiber gratings written in the two-mode fiber. Opt. Express 2016, 24, 6186-6195. [CrossRef] 
14. Fu, C.; Liu, S.; Wang, Y.; Bai, Z.; He, J.; Liao, C.; Zhang, Y.; Zhang, F.; Yu, B.; Gao, S.; et al. High-order orbital angular momentum mode generator based on twisted photonic crystal fiber. Opt. Lett. 2018, 43, 1786-1789. [CrossRef]

15. Fu, C.; Liu, S.; Bai, Z.; He, J.; Liao, C.; Wang, Y.; Li, Z.; Zhang, Y.; Yang, K.; Bin, Y.; et al. Orbital Angular Momentum Mode Converter Based on Helical Long Period Fiber Grating Inscribed by Hydrogen \& ndash; Oxygen Flame. J. Light. Technol. 2018, 36, 1683-1688. [CrossRef]

16. Fu, C.; Wang, Y.; Bai, Z.; Liu, S.; Zhang, Y.; Li, Z. Twist-direction-dependent orbital angular momentum generator based on inflation-assisted helical photonic crystal fiber. Opt. Lett. 2019, 44, 459-462. [CrossRef]

17. Ran, Z.L.; He, X.; Rao, Y.J.; Sun, D.; Qiu, X.J.; Zeng, D.B.; Chu, W.W.; Wei, Y.B. Fiber-Optic Microstructure Sensors: A Review. Photon. Sens. 2021, 11, 227-261. [CrossRef]

18. Mamidi, V.R.; Kamineni, S.; Ravinuthala, L.N.; Madhuvarasu, S.S.; Thumu, V.R.; Pachava, R.V.; Putha, K. Fiber Bragg gratingbased high temperature sensor and its low-cost interrogation system with enhanced resolution. Opt. Appl. 2014, 44, $299-308$. 